\title{
A Delta-Sigma beamformer with integrated apodization
}

Tomov, Borislav Gueorguiev; Stuart, Matthias Bo; Hemmsen, Martin Christian; Jensen, Jørgen Arendt

\section{Published in:}

Proceedings of SPIE

Link to article, DOI:

$10.1117 / 12.2006731$

Publication date:

2013

Link back to DTU Orbit

Citation (APA):

Tomov, B. G., Stuart, M. B., Hemmsen, M. C., \& Jensen, J. A. (2013). A Delta-Sigma beamformer with integrated apodization. In Proceedings of SPIE: Medical Imaging 2013: Ultrasonic Imaging, Tomography, and Therapy SPIE - International Society for Optical Engineering. https://doi.org/10.1117/12.2006731

\section{General rights}

Copyright and moral rights for the publications made accessible in the public portal are retained by the authors and/or other copyright owners and it is a condition of accessing publications that users recognise and abide by the legal requirements associated with these rights.

- Users may download and print one copy of any publication from the public portal for the purpose of private study or research.

- You may not further distribute the material or use it for any profit-making activity or commercial gain

- You may freely distribute the URL identifying the publication in the public portal 


\title{
A Delta-Sigma beamformer with integrated apodization
}

\author{
Borislav Gueorguiev Tomov, Matthias Bo Stuart, Martin Christian Hemmsen, \\ Jørgen Arendt Jensen
}

Center for Fast Ultrasound Imaging, Technical University of Denmark, 2800 Lyngby, Denmark

\begin{abstract}
This paper presents a new design of a discrete time Delta-Sigma $(\boldsymbol{\Delta} \boldsymbol{\Sigma})$ oversampled ultrasound beamformer which integrates individual channel apodization by means of variable feedback voltage in the Delta-Sigma analog to digital (A/D) converters. The output bit-width of each oversampled A/D converter remains the same as in an unmodified one. The outputs of all receiving channels are delayed and summed, and the resulting multi-bit sample stream is filtered and decimated to become an image line. The simplicity of this beamformer allows the production of high-channel-count or very compact beamformers suitable for 2-D arrays or compact portable scanners. The new design is evaluated using measured data from the research scanner SARUS and a BK-8811 192 element linear array transducer (BK Medical, Herlev, Denmark), insonifying a water-filled wire phantom containing four wires orthogonal to the image plane. The data are acquired using 12-bit flash A/D converters at a sampling rate of $70 \mathrm{MHz}$, and are then upsampled off-line to $560 \mathrm{MHz}$ for input to the simulated $\boldsymbol{\Delta} \boldsymbol{\Sigma}$ beamformer. The latter generates a B-mode image which is compared to that produced by a digital beamformer that uses 10-bit A/D converters. The performance is evaluated by comparing the width of the wire images at half amplitude and the noise level of the images. The $\Delta \boldsymbol{\Sigma}$ beamformer resolution has been found to be identical to that of the multi-bit A/D beamforming architecture, while the noise floor is elevated by approximately $6 \mathrm{~dB}$.
\end{abstract}

Keywords: Ultrasound imaging, Delta Sigma converter, beamforming

\section{INTRODUCTION}

Multi-bit Nyquist-rate analog-to-digital (A/D) converters are the core building blocks of modern digital ultrasound beamformers. Using Nyquist-rate A/D converters, significant effort has to be put into signal preconditioning and the generation of both voltage and time references. Oversampling A/D converters trade digital signal processing complexity for relaxed requirements on the analog components compared to Nyquist-rate A/D converters. The Delta-Sigma modulation (DSM) A/D converter combines both oversampling and noise shaping, and makes it possible to effectively convert an electrical analog signal into its digital, discrete-time representation using modern digital CMOS technology. ${ }^{1-3}$ The use of such converters in ultrasound beamformers offers the following advantages: integration of beamforming logic and A/D converters, as these can be manufactured using the same semiconductor technology; small area per converter, allowing to accommodate a large number of A/D converters on a single chip; and low price.

Direct use of DSM A/D converters, followed by first-in-first-out (FIFO) fixed-length delay buffers, adder and filter/decimator results in a simple fixed focus beamformer with great scalability. Unfortunately, such a beamformer lacks two features necessary for high quality imaging: weighting/apodization of the received signals, and dynamic adjustment of the relative delays between the channel data for the purpose of maintaining a shifting receive focus that follows the current reconstructed echo sample.

A number of researchers have worked on incorporating dynamic receive focusing in $\Delta \Sigma$ beamformers. ${ }^{4-6}$ The solutions have suffered to various degrees from introducing extra noise into the digitized signal and/or requiring additional digital logic. The need for dynamic receive focusing can be overcome by utilizing sequential synthetic aperture beamforming, ${ }^{7}$ where the first beamforming stage is a fixed-focus beamformer. With that application in mind, the current paper presents a comparison of fixed-focus beamformers.

Send correspondence to Borislav Gueorguiev Tomov: E-mail: bt@elektro.dtu.dk, Telephone: +45 45253901 
The problem of integrating channel apodization into such a beamformer has persisted until now. The obvious approach of using coefficient registers on each channel, selectable by the ADC output bit value (in a case of a bilevel quantizer), requires a large number of multi-bit adders, which counteracts the goal of simplicity/scalability of the beamformer.

Variable $\Delta \Sigma$ modulator feedback has been investigated by Norman ${ }^{8}$ for the purpose of implementing time gain compensation inside an oversampled converter. In that work, apodization across the channels was not considered.

This paper presents a new design of a discrete time Delta-Sigma $(\Delta \Sigma)$ oversampled ultrasound beamformer which integrates individual channel apodization by means of variable feedback voltage in the Delta-Sigma analog to digital $(\mathrm{A} / \mathrm{D})$ converters. The output bit-width of each oversampled A/D converter remains the same as in an unmodified one. The outputs of all receiving channels are delayed and summed, and the resulting multi-bit sample stream is filtered and decimated to become an image line. The involved signal processing principles are presented in Section 2. A comparison of the obtained image to one made using a 10-bit A/D based digital beamformer has been made, and the results are presented in Section 3. The discussion of the suggested new architecture is presented in Section 4, followed by conclusion in Section 5.

\section{METHODS}

This section introduces $\Delta \Sigma$ modulators with variable feedback gain and illustrates their use in a beamformer architecture that features channel weighting (apodization).

\subsection{Delta-Sigma modulation}

A $\Delta \Sigma$ modulator combines noise shaping and oversampling. A second order modulator uses two integration stages within a feedback path, as shown in Fig. 1, such that the quantizer output tracks the average input. The difference between quantizer output and modulator input accumulates in the integrators and ultimately corrects itself, so that the averages are identical. Apodization is integrated into the modulator by implementing a variable feedback voltage. In the current work, a bi-level quantizer (a comparator) was chosen. That is the simplest quantizer possible, and does not require precision manufacturing as multi-level quantizers do. Imperfection in its threshold level manifests itself as a DC bias in the A/D conversion output. In ultrasound imaging, bandpass matched filters are used, so any DC components are filtered out.

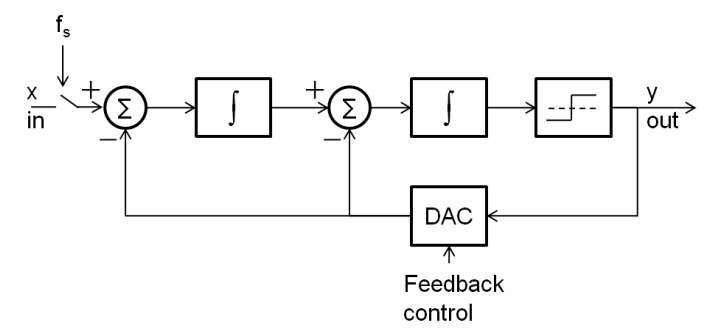

Figure 1. A discrete time second order $\Delta \Sigma$-modulator block diagram.

\subsection{Delta-Sigma Beamforming}

$\Delta \Sigma$ receive beamformers have three important benefits over conventional multi-bit methods. First, the A/D converters can be fabricated on the same chip as the processing circuitry creating the received scan lines. Thus, interconnect, size, and price are reduced. Second, accurate delays can be achieved simply by manipulating individual samples at the high sampling rate. Third, the sum across the array is performed on single bits. The block diagram of a $\Delta \Sigma$ based receive beamformer, as first proposed by Noujaim et al. ${ }^{9}$ and here modified to include variable feedback gain, $G$, is shown in Fig. 2. Fixed focus beamforming is achieved by fixing the output position of the FIFO buffers to cells whose indexes are calculated from the required inter-channel delays relative to the channel that has the shortest flight path to the receive focal point. These output positions are switched for each new emission in order to accommodate new receive focal point, corresponding to a new image line. 


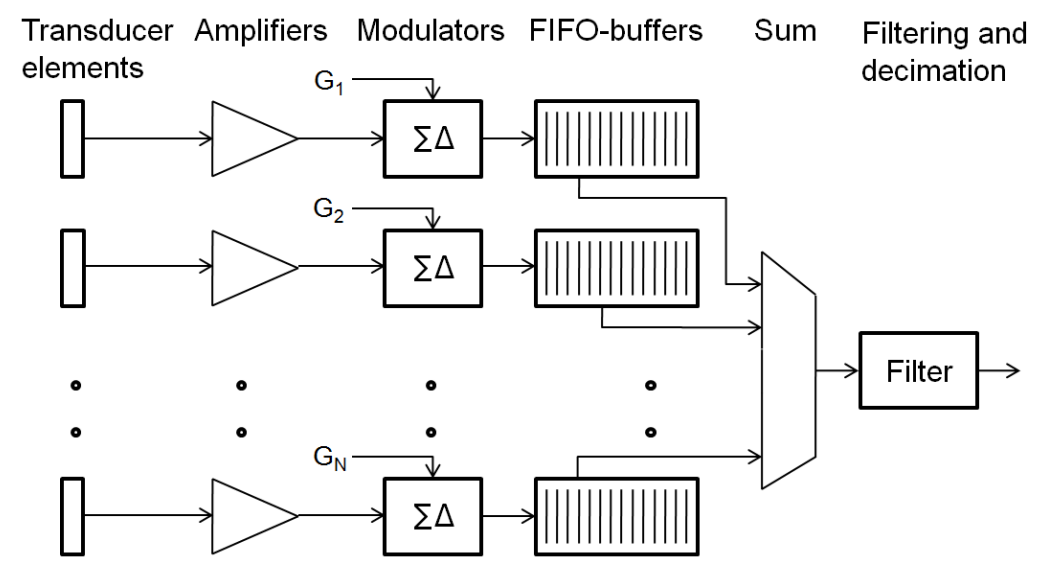

Figure 2. $\Delta \Sigma$ beamforming architecture implementing variable feedback gain for each individual receiving channel.

\subsection{Oversampling ratio calculation}

The following calculations are based on the assumptions that the individual channel noise components are not correlated between channels, the spectrum density of the analog noise is constant across the spectrum (at the input of the ADC), and the signal is a sinusoidal one.

The theoretical signal-to-noise ratio (SNR) of a multi-bit Nyquist-rate A/D converter is calculated (equation $\left.(11.16) \mathrm{in}^{10}\right)$ as

$$
S N R=6.02 \times N+1.76 \mathrm{~dB},
$$

where $\mathrm{N}$ is the number of output bits of the $\mathrm{A} / \mathrm{D}$ converter, and the possible levels to represent the input are $2^{N}$.

A 10-bit A/D converter, thus, achieves a SNR of

$$
S N R=6.02 \times 10+1.76=62 \mathrm{~dB} .
$$

The theoretical signal-to-noise ratio (SNR) of a second-order $\Delta \Sigma \mathrm{A} / \mathrm{D}$ converter is calculated (equation $(14.32)$ in $\left.^{10}\right)$ as

$$
S N R=6.02 \times N+1.76-12.9+50 \log \frac{f_{s}}{2 f_{0}} \mathrm{~dB},
$$

where $f_{s}$ is the sampling rate and $f_{0}$ is the frequency of the signal of interest. The fraction in the brackets is called oversampling ratio (OSR).

To achieve the 10-bit $\mathrm{A} / \mathrm{D}$ converter $\mathrm{SNR}$, a second-order $\Delta \Sigma \mathrm{A} / \mathrm{D}$ converter needs to operate at an oversampling ratio (after transforming Eqn. 3) of

$$
O S R=10^{\frac{62-6.02-1.76+12.9}{50}} \approx 22
$$

An ultrasound beamformer creates image lines by focusing in different directions by means of applying delays to the signals from individual elements, which are then summed. In doing that, the noise components in the input signals are also summed. Assuming uncorrelated noise components with root-mean-square (RMS) values of $V_{i}$, the sum noise component will have an RMS value (by extending equation (4.14) in ${ }^{10}$ ) of 


$$
V_{\text {sum }}=\sqrt{\sum_{i=1}^{64} V_{i}^{2}}
$$

As the echo data is summed across channels in an ultrasound beamformer, apodization is applied in order to reduce edge effects and improve the sidelobe level of the imaging system point spread functions. When doing that, the sampled noise (analog + quantization) components $V_{i}$ in the individual channels do not add with equal amplitudes, but with scaled ones: $V_{i}=c_{i} V_{n}$, where where $c_{i}$ are the windowing function coefficients. For the case of apodization using the Hamming windowing function, applied to 64 signals, the noise amplitude increase can be calculated as

$$
\frac{V_{n 64}}{V_{n}}=\sqrt{\sum_{i=1}^{64} c_{i}^{2}}=5,
$$

The increase of amplitude of the useful signal, assuming it has identical amplitude $V_{s}$ on all channels before the apodization, is

$$
\frac{V_{s 64}}{V_{s}}=\sum_{i=1}^{64} c_{i}=34.1,
$$

therefore the increase in SNR is $34.1 / 5=6.82$ times, equivalent to $6 \times \log _{2} 6.82=16.6 \mathrm{~dB}$. The resulting SNR after the channel signals sum is $62+16.6=78.6 \mathrm{~dB}$.

Using the built-in apodization capability in the oversampled converters, the noise components (analog + quantization) in their output will have the same amplitudes and the summed noise component will grow by

$$
\frac{V_{n 64 o}}{V_{n o}}=\sqrt{\sum_{i=1}^{64} 1^{2}}=8,
$$

The signal of interest will grow in the same way as in a multi-bit architecture (eqn. (7)). Thus, for 64 channels, the SNR improvement will be $6 \log _{2} \frac{34.1}{8}=12.6 \mathrm{~dB}$, and matching the SNR performance of the 10-bit A/D beamformer will require an oversampling ratio of

$$
O S R=10^{\frac{62+16.6-12.6-6.02-1.76+12.9}{50}}=26.4,
$$

which, for a $7 \mathrm{MHz}$ center frequency of the signal and assumed $100 \%$ bandwidth, results in a required sampling frequency for the Delta-Sigma modulators of $7 \times 1.5 \times 2 \times 26.4=554 \mathrm{MHz}$.

\section{MEASUREMENT RESULTS}

Raw radio frequency element data from a phantom containing four wires orthogonal to the imaging plane was acquired using the SARUS research scanner, ${ }^{11,12}$ and a linear array transducer (BK-8811, BK Medical, Herlev, Denmark). For generating the B-mode images, the scanner was set up to acquire 192 scan lines with a transmit focus set at a depth of $4 \mathrm{~cm}$, using 64 active transmit elements weighted by a Hamming window function. The excitation was a two-cycle sinusoid with $f_{0}=7 \mathrm{MHz}$. The receive beamforming was done using the same fixed delay profile as in transmit, using the same elements and apodization function.

Data was acquired at a sampling rate of $70 \mathrm{MHz}$, using 12-bit flash A/D converters. For use as an input to the $\Delta \Sigma$ beamformer, the measured data was offline upsampled to $560 \mathrm{MHz}$ (8 times upsampling). The combined decimation/matched filter was created by convolving the excitation to the impulse response of the transducer twice, and upsampling the result to the modulation frequency. The impulse response was assumed to be that of an ideal transducer (no tail/ringing) with $60 \%$ bandwidth around $7 \mathrm{MHz}$. The resulting FIR filter was quantized 


\begin{tabular}{lccccc} 
Wire index & Depth & Lat. width MB & Lat. width OS & Ax. width MB & Ax. width OS \\
\hline 1 & 33 & 1.04 & 1.04 & 0.49 & 0.49 \\
2 & 56 & 3.64 & 3.64 & 0.42 & 0.42 \\
3 & 79 & 5.98 & 5.98 & 0.44 & 0.43 \\
4 & 104 & 6.76 & 6.76 & 0.46 & 0.46
\end{tabular}

Table 1. Depth of phantom wires, and lateral and axial widths of the wire images in the multi-bit A/D beamformed (MB) and oversampled A/D beamformed (OS) images. All units are in mm.

to 10-bit representation and has length of 401 coefficients. The feedback level of the $\Delta \Sigma \mathrm{A} / \mathrm{D}$ converter was quantized to 16 levels (4-bit quantization) and the apodization in the multi-bit A/D beamformer was quantized to 256 levels (10-bit representation).

Fig. 3 shows the generated B-mode images by a conventional multi-bit beamformer and the $\Delta \Sigma$ beamformer, with $70 \mathrm{~dB}$ dynamic range. Fig. 4 shows the lateral and axial profiles of the wire images. Fig. 4 shows the lateral and axial profiles of the imaged wires. The widths of the wire images measured at $-6 \mathrm{~dB}$ in lateral and axial direction are presented in Table 3. As expected, the wires further away for the focal point have wider profiles.

As can be observed, there exists an interference pattern that has entered the sampled data. The noise level of the images under comparison was estimated by calculating the RMS value of the noise in an area that was relatively free of that pattern, at depths between 106 and $145 \mathrm{~mm}$ and lateral positions between -24.8 and -13.9 $\mathrm{mm}$. These values were then divided by the maximum signal values in the images, which in both cases are the clipped waveforms that represent the emitted signal. The estimated noise floor for the oversampled beamformer image was $-64.7 \mathrm{~dB}$ below maximum amplitude, and for the multi-bit beamformer it was at $-70.4 \mathrm{~dB}$ below maximum amplitude.

\section{DISCUSSION}

The two images exhibit the same resolution, therefore introducing apodization in the oversampled architecture has made its output indistinguishable from that of a multi-bit beamformer. The remaining drawback is the elevation of the noise floor, which is most likely due to non-optimal filtration for the purpose of removing the quantization noise.

\section{CONCLUSION}

A new $\Delta \Sigma$ beamforming architecture with integrated apodization in the discrete time $\Delta \Sigma$ modulator has been presented. The $\Delta \Sigma$ modulator implements apodization through variable feedback voltage. Using measured data and offline processing, it was shown that the $\Delta \Sigma$ beamformer focusing performance matches that of a conventional multi-bit beamforming architecture using 10-bit parallel $\mathrm{A} / \mathrm{D}$ converters, while the noise floor is elevated by about $6 \mathrm{~dB}$.

The presented architecture in this paper is expected to contribute to the further development of compact analog front-ends for multi channel scanners with very large channel count.

\section{Acknowledgments}

The presented work was financially supported by grant 024-2008-3 from the Danish Advanced Technology Foundation and by B-K Medical ApS, Denmark.

\section{REFERENCES}

[1] Inose, H., Yasuda, Y., and Murakami, J., "A telemetering system by code modulation - $\Delta \Sigma$-modulation," Space Electronics and Telemetry IRE Transactions on SET-8(3), 204-209 (1962).

[2] Candy, J. C. and Temes, G. C., [Oversampling Delta-Sigma Data Converters - Theory, Design and Simulation], IEEE Press (1992).

[3] Norsworthy, S. R., Shreier, R., and Temes, G. C., [Delta-Sigma Data Converters : Theory, design and simulation], Wiley-IEEE Press (1996). 

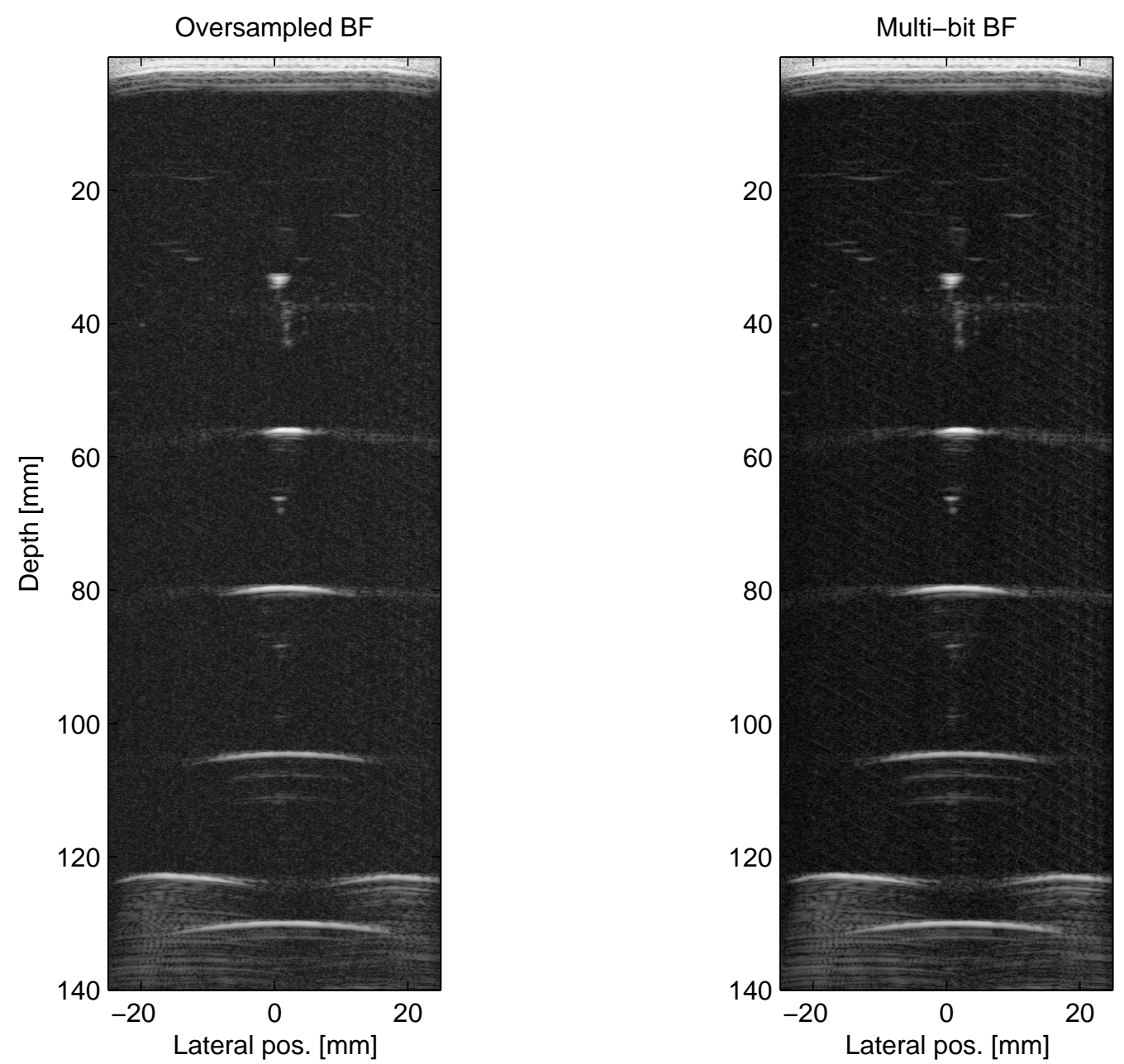

Figure 3. B-mode images generated using an oversampled beamformer (left) and a conventional beamformer (right), displayed with $70 \mathrm{~dB}$ dynamic range.

[4] Freeman, S. R., Quick, M. K., Morin, M. A., Anderson, R. C., Desilets, C. S., Linnenbrink, T. E., and O'Donnell, M., "Delta-sigma oversampled ultrasound beamformer with dynamic delays," IEEE Trans. Ultrason., Ferroelec., Freq. Contr. 46, 320-332 (1999).

[5] Kozak, M. and Karaman, M., "Digital phased array beamforming using single-bit delta-sigma conversion with non-uniform oversampling," IEEE Trans. Ultrason., Ferroelec., Freq. Contr. 48, 922-931 (2001).

[6] Tomov, B. and Jensen, J. A., "Compact FPGA-based beamformer using oversampled 1-bit A/D converters," IEEE Trans. Ultrason., Ferroelec., Freq. Contr. 52(5), 870-880 (2005).

[7] Kortbek, J., Jensen, J. A., and Gammelmark, K. L., "Sequential beamforming synthetic aperture imaging," Ultrasonics 53(1) (2013).

[8] Norman, O., "A band-pass delta-sigma modulator for ultrasound imaging at $160 \mathrm{MHz}$ clock rate," IEEE J. Solid-State Circuits 31, 2036-2041 (1996).

[9] Noujaim, S. E., Garverick, S. L., and O’Donnel, M., "Phased array ultrasonic beam forming using oversampled A/D converters." US Patent 5203335 (1993).

[10] Johns, D. and Martin, K., [Analog integrated circuit design], John Wiley \& Sons, Inc (1997).

[11] Jensen, J. A., Hansen, M., Tomov, B. G., Nikolov, S. I., and Holten-Lund, H., "System architecture of an experimental synthetic aperture real time ultrasound system," in [Proc. IEEE Ultrason. Symp.], 636-640 (Oct. 2007). 

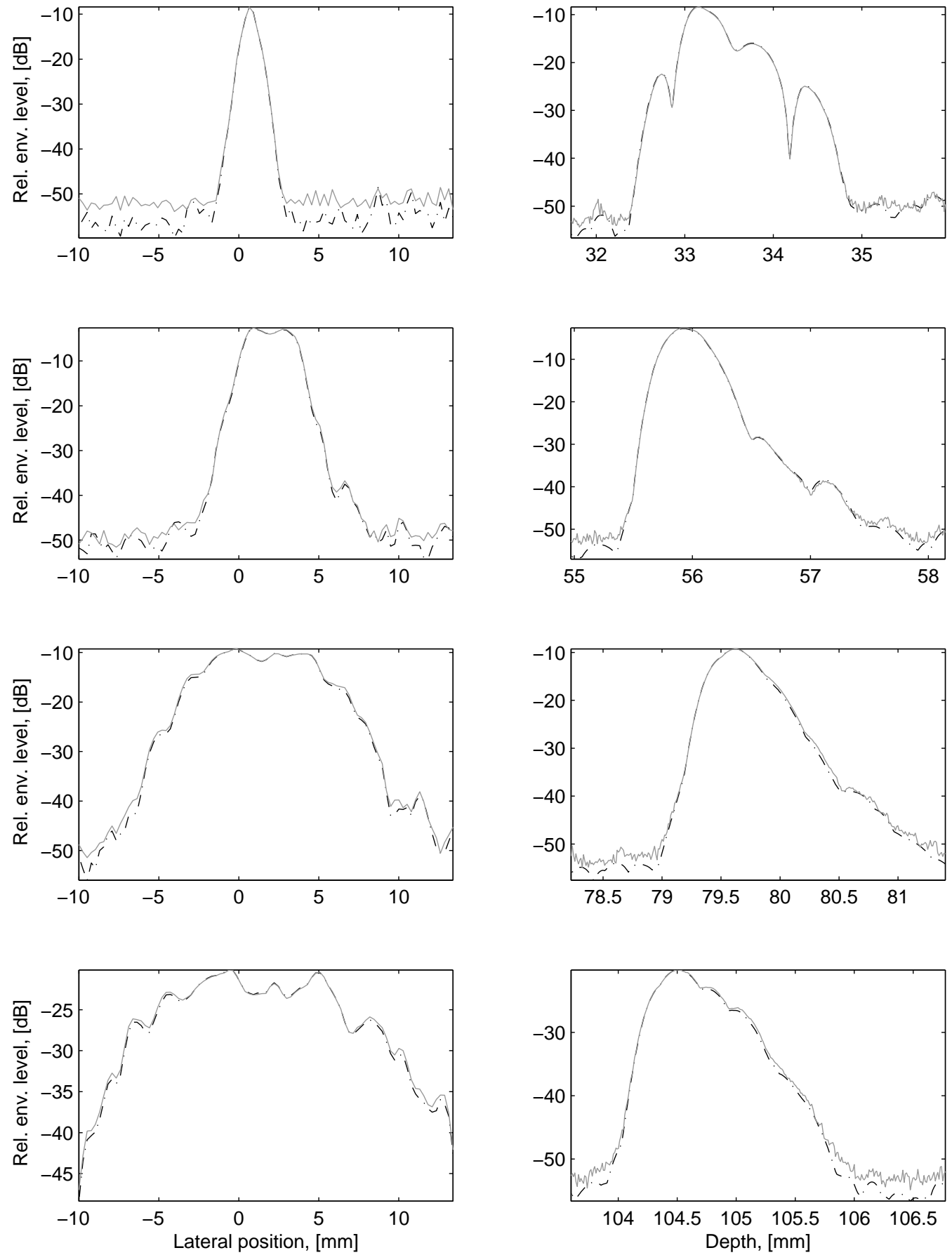

Figure 4. Lateral (left) and axial (right) profiles of the four image wires. The oversampled beamformer envelopes are shown in gray, and the multi-bit beamformer envelopes are shown with dash-dotted black line.

[12] Jensen, J. A., Holten-Lund, H., Nielson, R. T., Tomov, B. G., Stuart, M. B., Nikolov, S. I., Hansen, M., and Larsen, U. D., "Performance of SARUS: A Synthetic Aperture Real-time Ultrasound System," in [Proc. IEEE Ultrason. Symp.], 305-309 (Oct. 2010). 